\title{
Optimizing Call Drops in Cellular Network using Artificial Intelligence based Handover Schema
}

\author{
Avinash Singh ${ }^{1}$, Surya Pratap Singh ${ }^{1}$, Upendra Nath Tripathi ${ }^{2}$, Manish Mishra ${ }^{3}$ \\ Research Scholar, Department of Computer Science, D.D.U. Gorakhpur University, Gorakhpur, UP, India ${ }^{1}$ \\ Associate Professor, Department of Computer Science, D.D.U. Gorakhpur University, Gorakhpur, UP, India ${ }^{2}$ \\ Associate Professor, Department of Electronics, D.D.U. Gorakhpur University, Gorakhpur, UP, India ${ }^{3}$
}

\begin{abstract}
The heavy traffic load at peak time cause frequent congestion and call dropping in wireless cellular network which demands an intelligent way of handover management schemes for various calls in load balancing and resource sharing. The cellular network service provider currently facing certain obstacle in load balancing and resource sharing for mobile users. So for various call handover management schema that have been proposed for load balancing and sharing scheme are not efficient to minimizing the call drop in cellular communication. In this paper, we proposed an intelligent schema for load balancing and sharing in handover. The simulation results shows that the proposed scheme would able achieve satisfactory level in call drop optimization through handover management that concerning load balancing and sharing under heavy peak traffic load.
\end{abstract}

Keywords: Cellular network, call drop, call block, handoff, fuzzy logic, neuro-fuzzy logic.

\section{INTRODUCTION}

Presently several mobile subscribers were grappling with the arising issue of frequently call disconnections or "call drop' problem. Call drop is the common term used for describing any unexpected and unusual call termination of a wireless mobile call within cellular network. Recently, there has been wide incensement in the complaint from consumers regarding frequent call drops. While this call drop problem predominant in the subscriber base country where the mobile user are growing very fast, as compare to the mobile telecom infrastructure which is not growing at the same pace and immense pressure is being put on to the existing prior facilities, leads to a dip in the quality of services provided[1]. Call drop that affects the quality of expectation of the mobile subscribers, arises due to a variety of technical issues and challenges, including inadequate infrastructure; area coverage; signal quality \& strength; interference level; network congestion; and network failure. The remote area subscribers primarily face call drops because of lack of coverage, while in urban and metro areas; this arises due to the increasing gap between the increasing growth of mobile subscriber base and lack of investment in building network infrastructure to support the growing demand, including setting up of additional base transceiver stations (BTS) in given geographical area and establishment in-building lager coverage. Since the frequency reuse factor in which same type of frequency in a given cell could be reused within over different adjacent neighbour cell created limitation over radio resources without having call drop and handover problem [2].

Basically a cellular network subscriber owns certain few range of channel bands allocated by regulation authority's that could be frequencies, time slots or codes depending on the radio resource accessing techniques. Its allocated capacity often depend upon its total subscribers of service provider but network companies report more less than as actual demand know that only $25 \%$ user would be always active, but the load increases at peek time and that time call drop increased abruptly[3]. Arriving calls are accepted or rejected from access to the network by the call admit control rule based on the predefined policy [4]. When a user enters from one cellular network cell to other one, then call needs reallocation of channels having different frequency from previous one in this destination cell and this complete procedure of resource reallocation is termed as handoff process. In case of unavailability of resource in targeted cellular cell, this call forced for termination due to handoff procedure failure, which is highly unexpected at this stage. The potential performance measures in cellular networks are long term revenue, utility, call block rate (CBR) and call drop or handoff failure rate (CDR)[5]. CDR could be reduced by reserving some channel resources for handling incoming handoffs but, this reservation for CDR leads to stress on channel resources and increases in CBR. Hence, reduction effect of CBR and CDR are conflicting solution and optimizations of both are highly complex policy [6].

Various research have tried to solve this through the guard channel threshold policy, which a priori reserves a set of channels in each cell to handle handoff requests, is optimal for minimizing the CDR under specific conditions. But failed to handle the computational complexity of these approaches for high multi-class services having diverse characteristics scenario[7]. There are a variety of artificial 
Vol. 6, Issue 1, January 2017

intelligence techniques and fuzzy logic control (FLC) have been applied to the call admission control (CAC) schemes, but failed to handle [8]. This paper proposes a novel approach to solve the call drop problem in cellular networks with multiple classes of traffic with different resource and quality of services (QoS) requirements, and the traffic loads can vary according to the time. By prioritizing the handoff calls and load balancing schema for perceiving the real time CBR and CDR, the proposed learning scheme that would be trained by artificial learning and could be dynamically adjust loads with intelligently handling of handoff to minimize the CDR.

\section{RELATED WORK}

Call drop could be defined as a calls breaking due to technical issues, where conversation tear off before the communicating parties could have finalized their talks and before this, one of them has to hung up. A connected call might be terminated (disconnected) due to some technical problem before without prior permission ether participating parties to disconnect the ongoing call further leaving them into a hung up stage [10]. In resolving the problem relating modern cellular network issues most of the existing research is on call drop in cellular networks have focused on an abstract representation of the network in which only call admission control, (QoS) parameters, call block and drop solutions are considered.

A paper inspired from developing various mathematical approaches to solving the call admission control problem is described for the usage of Markov decision process, fuzzy logic, neural networks and genetic algorithms was introduced Which was trained through certain learning process able maintain automatically dynamically resource shearing between previous allocated resources and new instantaneous call request to achieve the desired level of preemption rate for new call[11] [12].

In this research paper proposed a fuzzy neural approach for call admission control in heterogeneous network having different class traffic and load for upcoming based new generation cellular network. This scheme combines the capabilities of both fuzzy logic linguistically control and neural network learning ability to reducing the call blocking possibility and increasing the resource utilization level [13].

The computational with artificial intelligence based use evolutional approach inspired from bio diversity such as the Genetic Algorithm (GA), Fuzzy Logic Control, Fuzzy Inference System (FIS) and Artificial Neural Networks (ANN). Major research work on computational and learning intelligence for call admission control algorithms involves fuzzy logic, fuzzy neural and neural network. But scantily of works have been reported on the usage of FLC or FIS with ANN base learning to consolidate on algorithms having prominent optimizations technique through an evolutionary and effective learning algorithm[14].

\section{III.PROPOSED CONCEPT OF SELF LEARNING FOR REDUCING CALL DROP RATE}

\section{A. Load Balancing And Sharing}

In cellular cell space any handover process is took place when the network signal strength of serving cell becomes week as compare to its network signal strength coming from neighborhood cell which signal strength is greater than threshold value and unable to maintain the ensured signal strength above threshold value that always be maintained is:

$$
\begin{gathered}
\text { RSStrength }_{\mathrm{j}}>=\text { RSStrengthMin }_{\mathrm{ij}} \\
\text { PESignal }_{\mathrm{ij}}=\text { RSStrength }_{\mathrm{j}}-\text { RSStrenth }_{\mathrm{i}}>=\text { HThreshol }_{\mathrm{i} . \mathrm{j}}
\end{gathered}
$$

Where RSStrength ${ }_{j}$ and RSStrenth $_{i}$ are the average received signal strength from neighbour cell $j$ and serving cell $i$, PESignal ${ }_{i \rightarrow j}$ is the paging expenditure of neighbour cell $j$ w.r.t. to serving cell $i, R \operatorname{SStrength} M i n_{i \rightarrow j}$ is the Handoff signal strength constraint and HThreshold $\mathrm{H}_{i \rightarrow j}$ is the handoff limit the adjacency.

\section{B. Fuzzy Logic Based New Call Admission Policy}

The proposed Fuzzy logic control, shown in the Figure 1, is influenced from diffusion based load balancing and sharing method [8]. The ambition is to reduction of CBR ratios in the targeted cellular cell. For this implementation in between handoff limit each pair of neighbour cells the rise in, Fuzzy logic control calculated by taking the difference of CBR between these neighbour cells and their current handoff margin values. By definition, the CBR is defined as:

$$
\text { CBR }=\text { Nblocked/Noffered }
$$

Where Nblocked is the no of blocked calls and Noffered overall number of offered calls in particular cell network respectively. The rise in handoff limit indicates through same magnitude but its sign shows direction of both neighbour cells signal strength with balance stability. However handoff limit are restricted to a limited variable time frame in order to avoid network fluctuation due to too much parameter changes. FLCs are based on the Takagi-Sugeno approach for optimization which is show in diagram with explanation.

The CBR difference between the two neighboring cells is feed as input to FLC. And for maintaining a balance between two close neighbor cells would be only achieved through continuously changing the hand off threshold limit. For adjusting the CBR balance between any two neighbor cells would be achieved through continuously changing the handoff limit of those close neighbors. By this way the whole CBR would be minimize in cellar network, as shown in Fig. 1 [3]. It is totally different from previous cases where it was quit sensitive matter for any slight changes would reflect as larger negative effect due involvements of too many parametric changes. Hence, one more FLC input in current handoff limit helps in reducing the magnitude and this slide change in threshold limit would convert it to negative. 
FLC inputs are classified according to linguistic terms by the fuzzifier. The fuzzifier translates input numbers into a value indicating the degree of membership to a linguistic term depending on a specific input membership function. For simplicity, the selected input membership functions are triangular or trapezoidal. In the inference engine, a set of if then else rules are defined to establish a relationship between the input and the output in linguistic terms. Finally, the defuzzifier obtains a crisp output value by aggregating all rules. The output membership functions are constants and the centre of gravity method is applied to obtain the final value of the output.

The given fuzzy scheme dynamically adjusts the guard bands to optimization level and while doing so it also achieves the system quality services and frequency resource utilization.

\section{Neuro-Fuzzy Reinforce Learning}

Reinforcement learning is an intelligent learning model based on trial and rewards (gain or loss) [9]. The basic concept behind reinforcement learning process is that how a learning system could be evolved to solve complicated tasks through repeated interactive process with the environmental interactions as given in figure 1. In a reinforcement learning process involves learning such that the state action set $\mathrm{Q}$ values ( $\mathrm{s}$, a), whose value would be the max-min discounted reward-penalties that could be achieved by initial step from state s, performing an action a, with following some optimal polices. The state action set that consists of discrete elements and having separate value exists for each course of action. Here the reinforcement learning implementation through neural network that would able solves the handoff problem of call drop in cellular network.

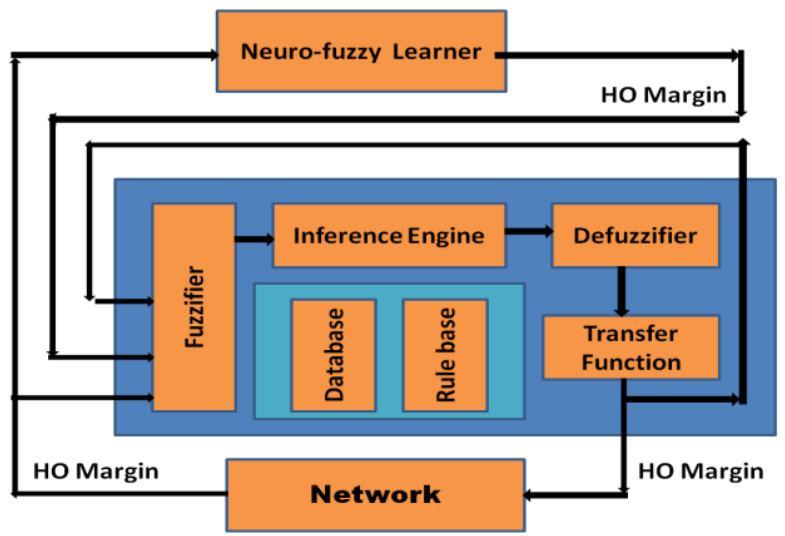

Fig. 1. Fuzzy Logic Control Based Design

At particular instant of time $t$, the agent chooses a course of action a from one of the state $\mathrm{s}$, the current set of state action pair elements values are calculated from (s, a) is donated by $\mathrm{Q}_{\mathrm{t}}(\mathrm{s}, \mathrm{a})$ is updated as follow:

$$
\begin{gathered}
Q_{t+1}(x, a)=Q_{t+1}(x, a)+\alpha\left[r_{t+1}+\gamma \operatorname{Max} Q_{t}(y, b)\right. \\
-Q(x, a)]
\end{gathered}
$$

b\&A y is the new next state. $0<\gamma<1$ is the reward/penalties factor and $\alpha$ is the rate of learning. Set A contains element for predicated course of actions and set $r$ outcome gain of agent when an action is chosen by him from moving to a new state $\mathrm{Q}_{\mathrm{t}+1}(\mathrm{~s}, \mathrm{a})$ from previous one. The state actions set value are calculated for some other states and with unchanged actions.

Fuzzy logic control reinforcement learning technique which evolves such course of action among these give set for selected rules that would help in maximize its adoptive learning process for incoming further new steps. The involving subscribers having different approaches for particular rule to apply on basis of previous experiences and certain situation those aeries which they have no option to react with a matching rule. In this case, consequents are equally distributed in the output interval. While exploring process the course of action among given set would be chosen such that would evaluate the max reinforcement $\mathrm{Q}$ value avoiding the min value leads to enriching the previous knowledge.

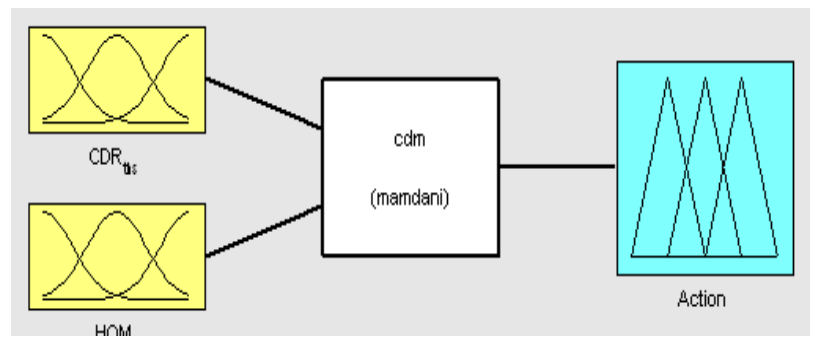

Fig. 2. Fuzzy Inference System (Mamdani FIS)

Dealing with multi-dimensional, continuous state spaces, in subject to utilization of involving function approximation discrete set values Q. under such situation most of the converging results concerning the reinforcement learning does not valid. Concerning approximation function we present a neuro-fuzzy logic scheme which involves the functional values having multilevel perceptions of neural network design. The prime focus on neural networks for this type problem which concerns the universal approximations and favouring best generalization capabilities. There is no direct allocation for the values to the element of set $\mathrm{Q}$ but these values are feed to involving functions which leads the final approximation.

Here, w denotes the neural network node's weights, a common way to approximate the optimal value function $\mathrm{Q}^{\mathrm{W}}(\mathrm{s}, \mathrm{a})$ is based on minimizing the error in equation: $\operatorname{minW} \Sigma_{(\mathrm{s}, \mathrm{a}) \varepsilon \mathrm{N}}\left(\mathrm{Q}^{\mathrm{W}}(\mathrm{s}, \mathrm{a})-\Sigma_{\mathrm{s}^{\prime}}\left(\operatorname{Psas}^{\prime}\left(\mathrm{n}(\mathrm{s}, \mathrm{a})+\min _{\left.\mathrm{bEA} A \mathrm{~s}^{\prime}\right)} \mathrm{Q}^{\mathrm{W}}\left(\mathrm{s}^{\prime}, \mathrm{b}\right)\right)^{2}\right.\right.$

where, $\mathrm{N}$ is a set of representing discrete state action elements Psas' and c(s, a) being evaluated through data.

To optimize the system of the FLC the logical variable is sets of call drop thresholds $\left(\mathrm{CDR}_{\mathrm{ths}}\right)$, handoff margins (HOM), course of action (Action) are determined by:

$$
\begin{aligned}
& \mathrm{V}\left(\mathrm{CDR}_{\mathrm{ths}_{\mathrm{th}}}\right)=\left\{\text { Unstabilized }_{\mathrm{i} \rightarrow \mathrm{j}}, \text { Stabilized, } \text { Unstabilized }_{\mathrm{j} \rightarrow \mathrm{i}}\right\} \\
& \mathrm{V}(\mathrm{HOM})=\{\mathrm{H}, \mathrm{M}, \mathrm{L}\} \\
& \mathrm{V}(\text { Action })=\{\mathrm{EL}, \mathrm{VL}, \mathrm{L}, \mathrm{N}, \mathrm{H}, \mathrm{VH}, \mathrm{EH}\}
\end{aligned}
$$


The chosen triangular functions as membership functions which balance and share the congested traffic load from for input and output logical variable are shown in Fig. 2, heavy to low leads minimize the call drop rate. Further, Fig. 3 and Fig. 4.

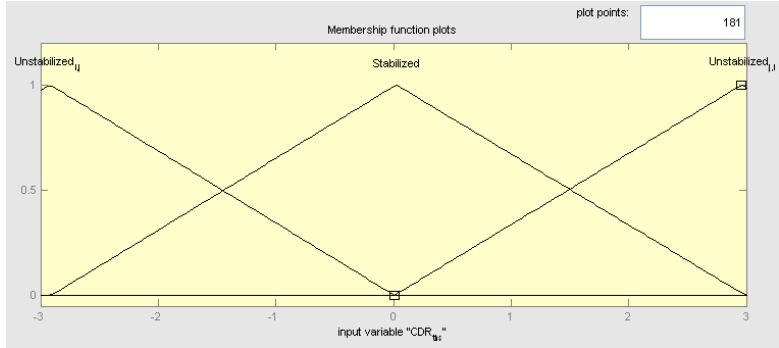

Fig. 3. Member Function for Call Drop Threshold $\left(\mathrm{CDR}_{\text {ths }}\right)$

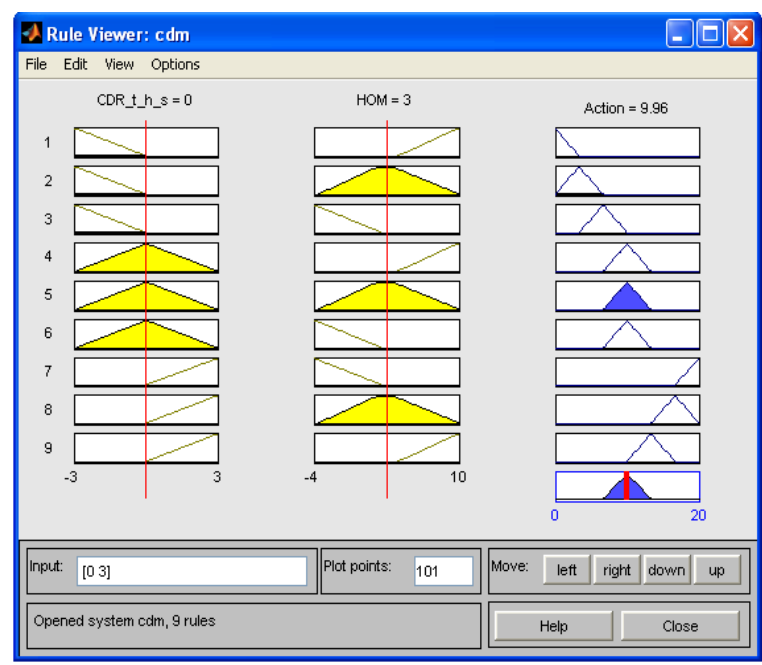

Fig. 4. Fuzzy Logic Rule Base

These logical values are passed to inference engine is connected with knowledge base which further link with database, rule base and output member functions. In knowledge base the database is to manipulate with fuzzy data which refined to obtain some essential definitions in describing the logical control variable.

\section{IV.SIMULATION AND RESULTS}

We have used real time learning capability simulator on Matlab tools. When simulation starts, it first load module with the arguments then after configure them and initialization with few entries. By iteration the simulator update mobile movement location, database computation, new call origination, resource utilization management process. Then after, analytical \& statistical output data and simulation result are recorded.

The simulation model is build of 19 hexagonal cell structures with non-uniformly distributed traffic load. Both uplink \& down link subscriber services like voice with low and high density load is considered which tries to share and balance the traffic load. Some cells having heavy traffic and other adjacent one have low traffic. We expects that the variable argument value being change with propagation of model through the fuzzy logic controller this process is learned dynamical with previous experience within real time through this neural network machine tool which helps us to bring call drop up to certain min level within very short duration, shown in Figure 6.

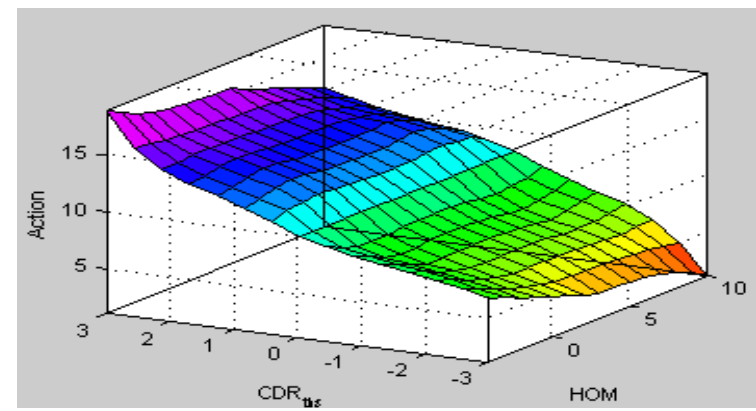

Fig. 5. Plot Between (CDR ths $)$ and (HOM)

For simulation when have chosen 60 hexagonal base station cell each having tri sector antenna and uneven distributed traffic density with random moves of cells. The adjustment plain, handoff arguments are symmetrically (18) and quarterly respectively. The linguistic fuzzy input and outputs terms with rules defined in fuzzy logic rule base Fig. 4.

And defined for the fuzzy input 'HO margin' are $L$ (low), $M$ (medium) and $H$ (high), while those defined for the fuzzy output ' $\triangle \mathrm{HO}$ margin' are $E L$ (extremely low), $V L$ (very low), $L$ (low), $N$ (null), $H$ (high), $V H$ (very high) and $E H$ (extremely high), which correspond to the crisp output values $-8,-4,-1,0,1,2,4$ and $8 \mathrm{~dB}$, respectively.

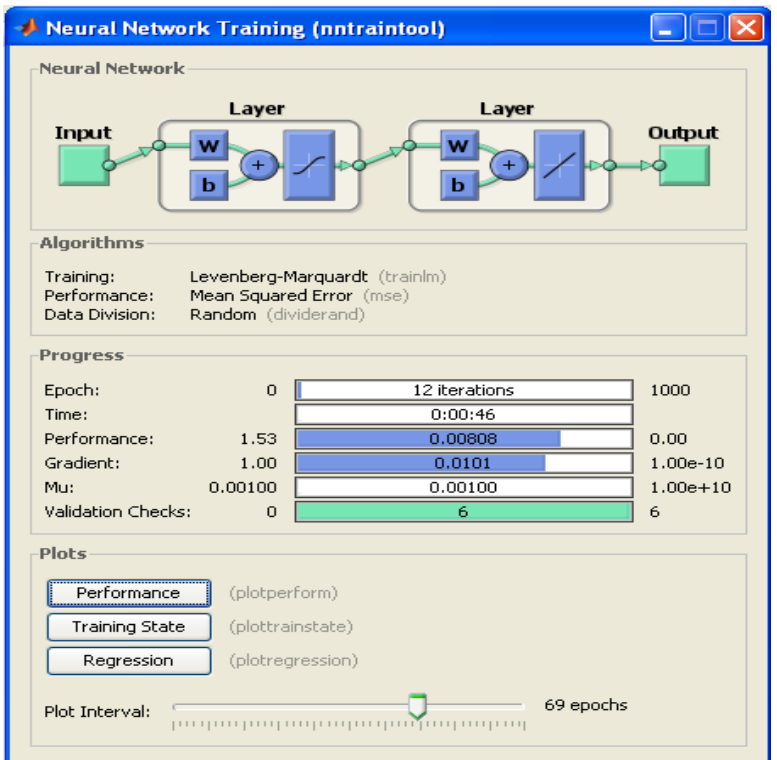

Fig. 6. Artificial Neural Network Based Intelligence Learning Tool

The Fig. 5 shows simulation graph of the call drop rate probability with changing traffic load of the given schemes. It also shows that the fuzzy logic based model has utilized the resources of in efficient way. 
Vol. 6, Issue 1, January 2017

\section{CONCLUSION}

This paper presents an optimization handoff technique that manage the call drop through dynamic load balancing \& sharing of resource sharing in real time quickly also have described an optimized FLC technique that have been tuned them dynamically. The optimization is achieved through neuro-fuzzy logic control system learning process which could enable the system to find best courses action that would helps in building rules for fuzzy inference engine. Then after the neuro-fuzzy learner enable this to learn at real time in short time duration.

The simulation graphs show the optimal situation could be achieved with significant reduction in call drop rate in highly congested load. The neuro-fuzzy logic based controller able to achieve the given task without modification in basic infrastructural design \& architecture. The insatiability arises due continuous handoff of traffic load sharing \& balancing problem is resolved by first exploration of right action by learning process and gets matured in limited duration through training.

\section{REFERENCES}

[1] TRAI, Technical Paper, "Call Drop in Cellular Networks", Telecom Regulatory Authority of India, on Mahanagar Door Sanchar Bhawan, J.L. Nehru Marg, New Delhi - 110 002, India, 2015.

[2] G. K. Verma, "Study and Survey on handoff failure in cellular Network and its minimization techniques", International Journal of Engineering and Technical Research (IJETR) ISSN: 2321-0869, Volume-2, Issue-12, December 2014

[3] Galadima Abednego, D. D. Danjuma and B. G. Buba, "The Analysis Of Inter Cell Handover Dynamics in A GSM Network", International Journal of Innovative Research in Science, Engineering and Technology, ISSN: 2319-8753, Vol. 3, Issue 6, June 2014.

[4] M. A. Khan, C. Truong, T. Geithner, F. Sivrikaya and S. Albayrak, "Network Level Cooperation for Resource Allocation in Future Wireless Networks", Technical University at Berlin, DAI-Labor, 10587 Berlin, Germany.

[5] A. K. Gangwar and V. Singh, "Quality of service improvement, Handoff Prioritization and Channel utilization for Cellular Network", Int. Journal of Engineering Research and Applications, ISSN: 2248-9622, Vol. 4, Issue 10 (Part-.2), pp.46-49, Oct. 2014

[6] X. Yang and J. Bigham, "A Call Admission Control Scheme using Neuro Evolution Algorithm in Cellular Networks", Int. J. Conf. on Artificial Intelligence, $20^{\text {th }}$ Int. J. Conf. Proc. on A.I., pp.186-189, Hyderabad, India, January 06 - 12, 2007.

[7] S. Arora1, S. Chaudhary and S. Solanki, "Automatically Optimization technique using a DNCL to avoid dropped calls in Cellular Network", International Journal of Advanced Research in Computer and Communication Engineering, ISSN (Print) : 23195940, ISSN (Online) : 2278-1021 Vol. 2, Issue 7, July 2013.

[8] G. N. Kumar and A. Arokiasamy, "An Efficient Combined Call Admission Control and Scheduling Algorithm to Improve Quality of Service for Multimedia Services Over Next Generation Wireless Networks", Department of CSE, Anjalai Ammal Mahalingam Engineering College, Kovilvenni, Tamil Nadu, India.

[9] J.C.H. Watkins and P. Dayan, Q-learning. Machine Learning, Vol.8: 279-292, 1992.

[10] S. S. Segeran and N. A. B. M. Radzi, "Determining the drop call rate, failed call rate and signal strength of Celcom mobile network in the University Tenaga National Putrajaya Campus", The 3rd National Graduate Conference (NatGrad2015), University Tenaga Nasional, Putrajaya Campus, 8-9 April 2015.
[11] I. Ahmad, J. Kamruzzaman, D. Habibi and F. Islam, “An Intelligent Model to Control Preemption Rate of Instantaneous Request Calls in Networks", Book- Ahead Reservation, IEEE, 978-1-4244-26034/08, 2008

[12] S. Kumar, A. Kumar and A. K. Pandey, "A Comparative Study of Call Admission Control in Mobile Multimedia Networks using Soft Computing", International Journal of Computer Applications, Volume 107 - No. 16, (0975-8887), December 2014.

[13] H.S. R. Babu, Gowrishankar and P.S. Satyanarayana, "An Intelligent Call Admission Control Decision Mechanism for Wireless Networks" Journal of computing, volume 2, Issue 4, ISSN 2151-9617, April 2010.

[14] P. Metre, K. Radhika and Gowrishankar, "Survey of Soft Computing Techniques for Joint Radio Resource Management", IEEE, 978-1-4673-1520, 2012.

\section{BIOGRAPHIES}

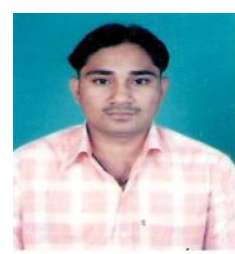

Avinash Singh is M.Sc. Computer Science, M. Tech and pursuing Ph.D. in the department of Computer Science. Deen Dayal Upadhyay Gorakhpur University, Gorakhpur (U.P. India) under the supervision of Dr. U.N. Tripathi. The area of research interest is Database Security, Networking. Mr. Avinash Singh has published 23 papers in different national and international conferences/ Journals.

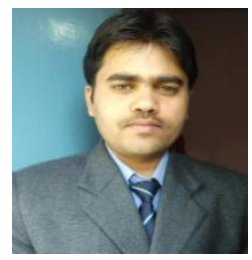

Surya Pratap Singh is MCA and UGC-NET qualified. He is pursuing Ph.D. In the department of Computer Science Deen Dayal Upadhyay Gorakhpur University, Gorakhpur (U.P. India) under the supervision of Dr. U.N. Tripathi. His area of research interest is Database Security, Algorithm design and Networking. Surya Pratap Singh has published more than 20 papers in different national and international conferences/ Journals.

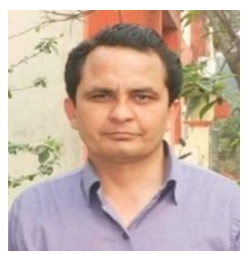

Dr. Upendra Nath Tripathi is Associate Professor in Department of Computer Science, Deen Dayal Upadhyay Gorakhpur University, Gorakhpur (U.P. India). He has 14 years of teaching and research experience. He has published more than 40 papers in various National and International Journals/conferences. His area of research interest is database systems and networking.

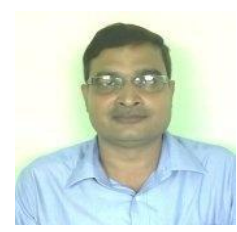

Dr. Manish Mishra is Associate Professor in Department of Electronics, Deen Dayal Upadhyay Gorakhpur University, Gorakhpur (U.P. India). He has 14 years of teaching and research experience. He has published more than 45 papers in various National and International Journals/conferences. His area of research interest is Computer Technology, fast processor design. 